\title{
ON EXTENDING ACTIONS
}

\author{
ROBERT VAUGHT
}

(Communicated by R. Daniel Mauldin)

\begin{abstract}
Consider a Polish topological group $G$ acting via $J$ on a substandard (= countably generated) Borel space. Theorem 1. Any such "Borel action" can be extended to a Borel action $J^{\prime}: G \times X^{\prime} \rightarrow X^{\prime}$ where $X^{\prime}$ is coanalytic. (Theorem 3 gives an analogue for continuous actions.) Corollary 2. The result "in any Borel action, orbits are Borel" implies the (well-known) result "all such orbits are absolutely Borel".
\end{abstract}

A Borel space is a set $Y$ together with a $\sigma$-field of subsets of $Y$. A Borel space $Y$ is called standard [resp. substandard] if it is isomorphic to the Borel structure of some Polish [resp. separable metrizable] topological space. If $Y$ is standard, $X$ is called an analytic subset of $Y$ if for some standard $Z$ and some $B$ Borel in $Y \times Z, X=\{y \in Y:(\exists z \in Z)(y, z) \in B\} . X$ is a coanalytic subset of $Y$ if the same holds with ' $\exists$ ' replaced by ' $\forall$ '. $X$ is called plain analytic [coanalytic] if it is an analytic [resp . coanalytic] subset of some standard space.

Suppose $G$ is a Polish topological group which acts on a substandard Borel space $X$ via a Borel map $J: G \times X \rightarrow X$. (Here "acts" means that for any $g, h \in G$ and any $x \in X, e x=x$ and $g(h x)=(g h) x$ where $g x=J(g, x)$.) Then $(J, G, X)$ is called a Borel action.

We will establish in Theorem 1 that any Borel action $(J, G, X)$ can be extended to a Borel action $\left(J^{\prime}, G, X^{\prime}\right)$ where $X^{\prime}$ is coanalytic.

Theorem 1 is applied in Corollary 2, which was its original motivation. In any Borel action $(J, G, X), \mathscr{O} \subseteq X$ is called an orbit if for some $x_{0} \in X$, $\mathscr{O}=\left\{g x_{0}: g \in G\right\}$. Douglas Miller [3] proved that in any Borel action $(J$, $G, X$ ), all orbits are Borel (in $X$ ). In so doing, he improved and extended various overlapping results of C. Kuratowski ([1, p. 377]), G. Mackey [1957] , J. Dixmier [1962 ], D. Scott [1964], C. Ryll-Nardzewski [1964], and this author [4]. (Missing references can be found in Miller [3].) In fact, Miller gave two proofs of his result. In one he used (among other things) cross-sections or selectors, as had Mackey, Dixmier, and Ryll-Nardzewski. In the other proof Miller extended to Borel spaces the "*-transform" method of the author [4] (which is loosely related to Scott's proof). Now, in fact, all of these authors

Received by the editors May 3, 1988. This material was presented to a Symposium on Descriptive Set Theory and Measure Theory at the AMS meeting in Atlanta, January 6-9, 1988.

1980 Mathematics Subject Classification (1985 Revision). Primary 54H15, 04A15. 
using cross-sections showed that all these orbits $\mathscr{O}$ are absolutely Borel (i.e., $\mathscr{O}$ is a Borel set in some standard space). On the other hand, the second proof only shows that each orbit in $(J, G, X)$ is a Borel set in $X$.

This situation is "resolved" in Corollary 2: The result that in every Borel action orbits are Borel implies that all such orbits are absolutely Borel.

$(J, G, X)$ is called a continuous action if $G$, as before, acts on a separable metrizable topological space $X$ via the continuous map $J: G \times X \rightarrow X$. We prove an analogue of Theorem 1 in Theorem 3: Any continuous action $(J, G$, $X)$ can be extended to a continuous action $\left(J^{\prime}, G, X^{\prime}\right)$, where $X^{\prime}$ is coanalytic (i.e., the Borel structure of $X^{\prime}$ is coanalytic).

\section{KNOWN FACTS}

A Borel space $X$ is Borel (resp. coanalytic) in some standard $Y$ if and only if $X$ is Borel (resp. coanalytic) in every standard $Z \supseteq X$ (cf. [2, §38, VII]).

Here are two well-known facts which can be found in stronger forms in [2, $\S 35, \mathrm{VI}]$, but are more easily just proved here.

Every substandard $X$ is isomorphic to a subspace of $2^{\omega}$.

Indeed, let $U_{1}, \ldots, U_{n}, \ldots$ be a set of $\sigma$-Boolean generators for the family of Borel sets of $X$. If $x \in X$, put $F(x)(n)=1$ if $x \in U_{n}, F(x)=0$ otherwise. One can now easily check that $F$ is a Borel isomorphism of $X$ onto $F[X]$, proving (1). (It follows that 'substandard' = 'subspace of standard'.)

$$
\begin{aligned}
& \text { Suppose } X_{2} \text { is substandard, } X_{1} \subseteq X_{2} \text {, and } F_{1}: X_{1} \rightarrow 2^{\omega} \text { is a } \\
& \text { Borel map. Then } F_{1} \text { can be extended to a Borel map } F_{2}: X_{2} \rightarrow \\
& 2^{\omega} \text {. }
\end{aligned}
$$

To see this put $U_{n}=\left\{x \in X_{1}: F_{1}(x)(n)=1\right\}$ for each $n$. Then $F: X_{1} \rightarrow 2^{\omega}$ and $U_{1}, \ldots, U_{n}, \ldots$ are as in the proof of (1). For each $n$, choose $U_{n}^{\prime}$ Borel in $X_{2}$ such that $U_{n}^{\prime} \cap X_{1}=U_{n}$. Define $F^{\prime}: X^{\omega} \rightarrow 2$ as $F$ was defined in the proof of (1) but now with respect to $X_{2}$ and $U_{0}^{\prime}, \ldots, U_{n}^{\prime}, \ldots$ It is easy to see $F^{\prime}$ is as desired.

The reader is referred to $[2, \S 35, \mathrm{I}$, Theorem 1], for the proof of

$\left(2^{\prime}\right) \quad$ Suppose $X_{2}$ is a separable, metrizable, topological space;

$X_{1} \subseteq X_{2}, Y$ is Polish, and $F: X_{1} \rightarrow Y$ is continuous. Then

$F$ can be extended to a continuous map $F^{\prime}: W \rightarrow Y$, where

$W$ is $a G_{\delta}$ subset of $X_{2}$.

\section{EXTENDING Borel ACTIONS}

Theorem 1. Any Borel action $J: G \times X \rightarrow X$ can be extended to a Borel action $J^{\prime}: G \times X^{\prime} \rightarrow X^{\prime}$, where $X^{\prime}$ is coanalytic.

Proof. By (1), $X$ is a subspace of an isomorph $Y$ of $2^{\omega}$. Thus $J: G \times X \rightarrow Y$ and $G \times X \subseteq G \times Y$. By (2), $J$ can be extended to a Borel map $J^{\wedge}: G \times Y \rightarrow Y$. 
( $J^{\wedge}$ need not be an action; however, we still write $g y=J^{\wedge}(g, y)$. ) Put

$$
X^{\prime}=\{y \in Y: e y=y \wedge(\forall g, h \in G)(g(h y)=(g h) y)\} .
$$

Clearly, $X \subseteq X^{\prime}$. Since $Y$ and $G$ are standard while $J^{\prime}$ and group multiplication are both Borel maps, it is easy to check that $X^{\prime}$ is coanalytic in $Y$.

Suppose $x^{\prime} \in X^{\prime}$ and $k \in G$. We claim that $k x^{\prime} \in G^{\prime}$. Indeed, since $x^{\prime} \in X^{\prime}, e\left(k x^{\prime}\right)=(e k) x^{\prime}=k x^{\prime}$. Also, for any $g, h \in G$, since $x^{\prime} \in X^{\prime}$, we have $g\left(h\left(k x^{\prime}\right)\right)=g\left((h k) x^{\prime}\right)=(g(h k)) x^{\prime}=((g h) k) x^{\prime}=(g h)\left(k x^{\prime}\right)$. Thus $k x^{\prime} \in X^{\prime}$, as claimed.

Now let $J^{\prime}=J^{\wedge} \mid\left(G \times X^{\prime}\right)$. Since $X \subseteq X^{\prime}, J^{\prime}$ extends $J$. As we just proved, $J^{\prime}: G \times X^{\prime} \rightarrow X^{\prime}$, Clearly $J^{\prime}$ is a Borel map. The conditions $e x^{\prime}=x^{\prime}$ and $g\left(h x^{\prime}\right)=(g h) x^{\prime}$ (for all $x^{\prime} \in X^{\prime}$ ) were built into the definition of $X^{\prime}$. Thus $\left(J^{\prime}, G, X^{\prime}\right)$ is a Borel action extending $J$ with $X^{\prime}$ coanalytic, and Theorem 1 is proved.

\section{Application to orbits}

Corollary 2. The result that in every Borel action, all orbits are Borel, implies that all such orbits are absolutely Borel.

Proof. Assume that in all Borel actions, orbits are Borel. Let $\mathscr{O}$ be an orbit in a Borel action $(J, G, X)$. Then $(G, J \uparrow(G \times \mathscr{O}), \mathscr{O})$ is also a Borel action. Thus (as was long known) it is enough to consider only the case $\mathscr{O}=X$.

By Theorem 1 we can extend $J$ to a Borel action $J^{\prime}: G \times X^{\prime} \rightarrow X^{\prime}$ where $X^{\prime}$ is a coanalytic subset of some standard $Y$. As was also long known, $\mathscr{O}=X$ is an analytic subset of $Y$. (Indeed, if $x_{0} \in \mathscr{O}$ then $\mathscr{O}=\{x \in Y:(\exists g \in G)$ $\left.\left(x=J\left(g, x_{0}\right)\right)\right\}$.) It follows easily that $\mathscr{O}$ is an analytic subset of $Y$. On the other hand, $\mathscr{O}$ is clearly an orbit in the action $\left(J^{\prime}, G, X^{\prime}\right)$, so $\mathscr{O}$ is Borel in $X^{\prime}$, by our general assumption. Since, in turn, $X^{\prime}$ is coanalytic in $Y$, it follows very easily that $\mathscr{O}$ is coanalytic in $Y$. Thus $\mathscr{O}$ is both analytic and coanalytic in $Y$; so by Souslin's Theorem $([2, \S 39$, III, Cor . 1]), $\mathscr{O}$ is Borel in $Y$. Hence, since $Y$ is standard, $\mathscr{O}$ is absolutely Borel, as was to be proved.

\section{EXTENDING CONTINUOUS ACTIONS}

Theorem 3. Any continuous action $(J, G, X)$ can be extended to a continuous action $\left(J^{\prime}, G, X^{\prime}\right)$ where $X^{\prime}$ is coanalytic.

Proof. The separable metrizable space $X$ is a subspace of some Polish space $Y$ (using the "completion"). Thus $J: G \times X \rightarrow Y$ is continuous and $G \times X \subseteq$ $G \times Y$. Hence, by $\left(2^{\prime}\right), J$ can be extended to a continuous map $J^{\wedge}: W \rightarrow Y$ where $W$ is a $G_{\delta}$ subset of the Polish space $G \times Y$. It follows that $W$ is Polish (cf. [2, §33, VI]). Write $g y$ for $J^{\wedge}(g, y)$ (which may not exist). Put

$$
\begin{gathered}
X^{\prime}=\{y \in Y:(\mathrm{A})(\forall h \in G)(h y \text { exists, i.e., }(h y) \in W) \wedge(\mathrm{B}) e y=y \wedge \\
\wedge(\mathrm{C})(\forall g, h \in G)(g(h y) \text { exists and }=(g h) y)\} .
\end{gathered}
$$


Clearly $X \subseteq X^{\prime}$. It is easy to check that $X^{\prime}$ is coanalytic in $Y$ - hence coanalytic. (As an example of the arguments needed, we show that $Y_{1}=\{y \in$ $Y: e y$ exists and $=y\}$ is Borel in $Y$. Clearly $Y_{2}=\{y \in Y:(e, y) \in W\}$ is Borel in $Y$. Also $y \in Y_{2} \mapsto J^{\wedge}(e, y)$ is a Borel map of $Y_{2}$ to $Y$. Hence $Y_{1}=\left\{y \in Y_{2}: J^{\wedge}(e, y)=y\right\}$ is Borel in $Y_{2}$, and hence in $Y$.) Also, by (A), $G \times X^{\prime} \subseteq W$. Put $J^{\prime}=J^{\wedge} \uparrow\left(G \times X^{\prime}\right)$. Clearly $J^{\prime}: G \times X^{\prime} \rightarrow Y$ and $J^{\prime}$ is continuous.

Let $x^{\prime} \in X^{\prime}$ and $k \in G$. We claim that $k x^{\prime} \in X^{\prime} \quad\left(k x^{\prime}\right.$ exists by (A)). First we show (A) holds (for $\left.y=k x^{\prime}\right)$. Let $h \in G$. By (C) (for $\left.y=x^{\prime}\right), h\left(k x^{\prime}\right)$ exists, as desired. Next we want to see that (B) holds (for $y=k x^{\prime}$ ). But by (C) (for $\left.y=x^{\prime}\right), e\left(k x^{\prime}\right)$ exists and $=(e k) x^{\prime}=k x^{\prime}$, as desired. Finally, we show (C) holds (for $y=k x$ ). Let $g, h \in G$. By (C) (for $\left.y=x^{\prime}\right), g\left((h k) x^{\prime}\right)$ exists and $=(g(h k)) x^{\prime}=((g h) k) x^{\prime}=($ by $(C))(g h)\left(k x^{\prime}\right)$. Also by $(\mathrm{C}), h\left(k x^{\prime}\right)$ exists and $=(h k) x^{\prime}$; hence $g\left(h\left(k x^{\prime}\right)\right)$ exists and $=g\left((h k) x^{\prime}\right)$, which $=(g h)\left(k x^{\prime}\right)$ by the previous sentence. Thus (C) holds for $y=k x^{\prime}$.

It only remains to show that $J^{\prime}$ is an action. But this has been built into the definition of $X^{\prime}$. Thus $\left(J^{\prime}, G, X^{\prime}\right)$ is a continuous action extending $J$, with $X^{\prime}$ coanalytic, and Theorem 3 is proved.

Remark. In Theorems 1 and 3 (but not Corollary 2), the hypothesis that $G$ is a Polish topological group can be replaced by the assumption that $G$ is an analytic Borel group. The proofs above only need obvious changes.

\section{REFERENCES}

1. C. Kuratowski, Topologie I, $2^{i e ̀ m e}$ édition, Panstwowe Wydawnictwo Naukowe, Warsaw and Wroclaw, 1948.

2. __ Topology, Vol. 1, Panstwowe Wydawnictwo Naukowe (Warsaw) and Pergamon Press (New York, Oxford), 1966.

3. D. Miller, On the measurability of orbits in Borel actions, Proc. Amer. Math. Soc. 63 (1977), pp. $165-170$.

4. R. Vaught, Invariant sets in topology and logic, Fund. Math. 82 (1974), pp. 269-294.

Department of Mathematics, University of California, Berkeley, California 94720 